* National Research Fillow in Chemistry.

1 Kassel, L., article to appear soon in J. Phys. Chem.

2 Rice, O. K., and Ramsperger, H. C., J. Amer. Chem. Soc., 49, 1617 (1927).

${ }^{3}$ See, for example, Tolman, Statistical Mechanics with Application to Physics and Chemistry, Chemical Cat. Co., New York, 1927, p. 129.

4 This evidently gives only that part of the preceding volume for which $p_{1}{ }^{2}+q_{i}{ }^{2}$ is at least equal to $\epsilon_{0}$. The same sort of integration will be made repeatedly in this paper.

5 The $\mu$ weight for any state of a single oscillator is $h$; we extend the summation from $m=m$ to $m=n$, since we want all states in which the single oscillator has at least $m$ quanta.

- See, for example, Laska, W., Sammlung von Formeln der reinen und angewandten Mathematik, Vieweg und Sohn, Braunschweig, p. 15. But the proof is very easily given by induction.

7 The integral gives the contribution to the product of the classical oscillators; this is multiplied by the quantum contribution, and the sum taken over all possible total energies for the quantum oscillators.

- The expression following the first $h$ is the total $\mu$ weight for $s$ classical oscillators and $r-1$ quantum oscillators, with total energy between $\epsilon-q h \nu$ and $\epsilon-g h \nu+d \epsilon$.

- To get this, we multiply the contribution of $r$ quantum oscillators with $p$ quanta by that of $s$ classical oscillators with energy between $\epsilon-p h \nu$ and $\epsilon-p h \nu+d \epsilon$ and at least $\epsilon_{0}$ in a single oscillator, and sum over all possible values of $p$.

\title{
THE LIFE OF ATOMIC STATES AND THE INTENSITY OF SPECTRAL LINES
}

\section{By I. S. . BOwEN}

Norman Bridge Laboratory of Physics, California Institute of Thehnology

Communicated November 21, 1927

It has recently been possible to explain most of the strong nebular lines as lines arising from electron jumps from metastable states in oxygen and nitrogen. ${ }^{1}$ In the nebulae the emission of spectral lines can be observed under conditions of much higher rarefaction than can be found in any other terrestrial or astronomical source. Since these nebular lines occur only under these conditions, this very definitely indicates that the electron jumps causing them take place spontaneously without the interference of outside fields due to other ions, that can often be used to explain jumps of this sort when they are observed in terrestrial sources. This constitutes then the first direct evidence that metastable states are not absolutely metastable but are states of long mean life, i.e., states from which the probability of a spontaneous jump in unit time is very small.

Further evidence for this viewpoint is found in the observation by Rayleigh ${ }^{2}$ that the forbidden mercury line ${ }^{1} S-{ }^{3} P_{2}$ at $2270 \AA$ is absorbed about $1 / 1,000,000$ as strongly as the ${ }^{1} S-{ }^{3} P_{1}$ line at $2536 \AA$. Since there 
is a slight probability of absorption there must also be a slight probability of emission as well. In this experiment no ions were present although, since the pressure was high, the field due to the electric dipoles in other atoms might have been appreciable.

As was pointed out in the discussion of the nebular spectrum, this viewpoint makes it possible to explain the enormous variation in intensity of the nebular lines in going from terrestrial sources, where the lines are too weak to be observed, to the nebulae, where they are often the strongest lines in the spectrum. The intensity of the line depends directly on the ratio of the probability of the return of an atom from a higher state to a lower state with emission of that line to the sum of the probabilities of its return by all processes combined. Since the mean life of any given process is usually defined as the reciprocal of its probability in unit time, it is evident that the intensity of the line depends primarily on the ratio of the mean time between collisions of the second kind with other atoms or with the wall to the mean life of the state before spontaneous emission. If this ratio is small the majority of the atoms are taken out of the excited state by collisions of the second kind rather than by the emission of radiation and the line is weak. If, on the other hand, the ratio is large the atoms can radiate spontaneously without interference and the line appears strongly.

These same differences in lifetime can doubtless be used to explain various anomalous intensities found, to a lesser degree, in terrestrial sources. For example, calculations on the basis of the correspondence principle show that the intercombination jumps; i.e., jumps between a singlet and a triplet term, etc., in the simplest atoms are very improbable, or, interpreted on the above basis, have a very long life. In the case of the heavier atoms such as mercury the perturbations due to the other electrons are so great that the intercombination jumps have nearly the same probability per unit time as the regular jumps. The $2536 \AA$ line in mercury which is caused by an intercombination jump is one of the strongest lines. Even for this line, however, $\mathrm{Wien}^{3}$ finds a mean life before emission nearly five times as long as the life of the other mercury lines.

On going to the lighter elements such as magnesium, however, the intercombination line ${ }^{1} S_{-}{ }^{3} P_{1}$ at $4571 \AA$ is found to be quite weak even though it is the true resonance line of neutral magnesium. This is at once explicable if the low probability of this jump in unit time is interpreted as indicating a mean life so long that the majority of the atoms are returned by collisions of the second kind rather than by radiation.

In beryllium the corresponding line has never been observed although it has been carefully searched for under low-temperature furnace conditions that are known to be especially favorable for bringing out these resonance lines. ${ }^{4}$ This can be explained as due to a still lower probability 
per unit time and longer life, even comparable perhaps with that of the nebular lines.

This indicates a whole gradation of mean lives extending from that of the regular lines whose mean life before emission is of the order of $10^{-8}$ seconds to that of the nebular lines whose mean life before emission must be of the order of a second or more.

${ }^{1}$ Bowen, Nature, 120, 473, 1927; Pub. A. S. P., 39, 295, 1927.

${ }^{2}$ Rayleigh, Nature, 120, 295, 1927.

${ }^{3}$ Wien, Ann. Phys., 73, 483, 1924.

${ }^{4}$ A. S. King, unpublished results.

THE SPECTRA OF SINGLY AND DOUBLY IONIZED GERMANIUM (GE II AND III)

BY R. J. LANG

Department of Physics, University of Alberta

Communicated December 8, 1927

In a paper appearing in 1924, Lunt ${ }^{1}$ has given the measurements and intensities of the germanium lines appearing in the spectrum from a discharge tube containing $\mathrm{Ge}^{\mathrm{Cl}_{4}}$ and also the metallic spark in air from $3937 \AA$ to $7147 \AA$. The measures of Exner and Haschek from $4744 \AA$ to about $2200 \AA$ for the arc and spark are also available in Kayser's "Handbuch de Spectroscopie," Vol. V. Lunt has also placed the lines in three classes. In class I are those arc and spark lines which are not suppressed by self-induction; in class II are lines which are strong when self-induction is used in the metal spark in air but vanish completely when self-induction is eliminated, while in class III are given what the author calls "enhanced" lines. Lunt also gives a list of lines of common frequency separations in which, among others, is found three pairs having the common separation 360 and placed in class $I$.

In connection with the work here reported the metallic spark has been photographed by the author on the two-meter grating from $2000 \AA$ to $4000 \AA$ in air, from $1250 \AA$ to $2400 \AA$ in hydrogen and from $400 \AA$ to $4000 \AA$ in vacuum. The grating used was ruled, however, for the Lyman region and gives nothing but the stronger lines in the longer wave-lengths above about $3000 \AA$. For the intensity in the vac spark of certain lines longer than $4000 \AA$ and for the wave-length of those (see * Table 2) the author is indebted to Prof. Stanley Smith of this laboratory.

I. The Spectrum of Singly Ionized Germanium.-The ordinary series spectrum of Ge II will consist, according to the displacement law, of doub- 